\title{
SPIN EVOLUTION AND SATURATION: NEW INSIGHTS THROUGH 3D MHD SIMULATIONS OF YOUNG SOLAR ANALOGS
}

\author{
V. Réville ${ }^{1}$ and A.S. Brun ${ }^{1}$
}

\begin{abstract}
We examine how 3D MHD simulations can deliver clues on the mechanisms at the origin of angular momentum loss saturation of rapidly rotating solar-like stars. Based on a study of six targets, whose magnetic field has been observed by Zeeman Doppler Imaging (ZDI), we find that the saturation could be explained by a extremely strong coverage of the stellar surface of a large scale dipolar mode, in disagreement with recent works.
\end{abstract}

\section{Introduction}

Stellar activity, which includes many chromospheric and coronal diagnostics, has been often used as a proxy for the level of magnetic activity, and thus for internal dynamo processes that maintain the magnetic fields of solar-like stars. X-ray observations of the Sun show that these emissions come mostly from closed coronal loops, whose energy is related to the size of the structure, thus covering many different scales (see, e.g. Rosner et al. 1978). The average level of X-ray flux seems, however, to be mostly a function of two parameters, the rotational period and the color (or mass) (Noyes et al. 1984; Pizzolato et al. 2003), that can be combined in one: the Rossby number. Observations show a close to linear relationship between the $\mathrm{X}$ luminosity $L_{X}$ and the inverse Rossby $R o^{-1}$ up to a saturation level beyond which $L_{X}$ becomes constant. This saturation at low Rossby numbers (Pizzolato et al. 2003), has been associated with a change of dynamo regime, leading to either a saturation of the magnetic field amplitude or a drastic change in the magnetic geometry (or topology).

Similarly, the study of stellar rotation has identified the need for a regime change in the angular momentum loss of young stars. Barnes (2003) proposed that the observed gap in young clusters color-period diagram could be explained by a switch from a convective dynamo to a interface dynamo, defining the $\mathrm{C}$ and $\mathrm{I}$

${ }^{1}$ Laboratoire AIM Paris-Saclay, CEA Orme des Merisiers, 91191 Gif-sur-Yvette, France 
sequences. Brown (2014) developed this hypothesis invoking a stochastic change in the rotational braking of stars, possibly due to a topology change of the stellar magnetic field. In effect, stellar rotational braking on the main sequence is the consequence of the ejection of a magnetized stellar wind. Weber \& Davis (1967) showed the the Alfvén radius, where the accelerating wind reaches the Alfvén speed, acts as a lever arm on the angular momentum loss. As the magnetic topology controls the radial decay of the stellar magnetic field, it has been implied for years that a more complex topology than a simple dipole would lead to a less efficient braking (Barnes \& Sofia 1996; Kawaler 1988; Matt et al. 2012). However, a precise quantification of this effect has only been brought recently by Réville et al. (2015a). This work, as well as the work of Garraffo et al. (2015), suggests that a switch from a pure dipole to a pure high order multipole, would be able to explain the observed change in angular momentum loss.

Nonetheless, observations of stellar magnetic fields through Zeeman Doppler Imaging struggle to find a clear trend towards more complex topologies as the stellar rotation increases (Donati \& Landstreet 2009). As we shall see in the next section, we find in young stars' magnetic field strong dipolar components, which clearly dominates the braking over higher order structures. Besides, stellar dynamo theory and numerical simulations do not predict any dipole vanishing (Brun et al. 2013), which is necessary in the topology induced change of braking regime. Fortunately, the observational data can be reproduced with a more continuous process, where the angular momentum loss first varies linearly with rotation and then as the rotation rate cubed. Many spin evolution models (Bouvier et al. 1997; Matt et al. 2015) have introduced a saturation rotation rate where the regime change occurs, that depends on mass. This saturation is most likely of magnetic origin, and has as such been associated with the X-ray flux saturation, even though their precise relationship remain unclear: while the X-ray luminosity is controlled by the dynamics of closed regions, the angular momentum loss is a function of the escaping mass and magnetic flux coming from coronal holes (Réville et al. 2015a).

In Réville et al. (2016) we published a set of 3D simulations of young stars' wind, constrained by spectropolarimetric observations of the surface magnetic field. Interestingly, the computed angular momentum loss shows sign of saturation. Although very few stars are concerned -at most two out of six targets-, this work suggest that this saturation comes from the very important coverage of the stellar surface by close magnetic loops. One of the originality of this work is that, the closed loops are not organized in sunspots at small scale, but rather as a very large scale dipole. Moreover, the magnetic amplitude keeps increasing with rotation.

\section{Numerical simulation of stellar wind versus age and rotation}

Unlike the solar wind, whose properties have been strongly constrained over the past 30 years, stellar winds remain difficult to observe. Wood et al. (2005) have provided constraints on the mass loss of a dozen of solar-like stars, providing 


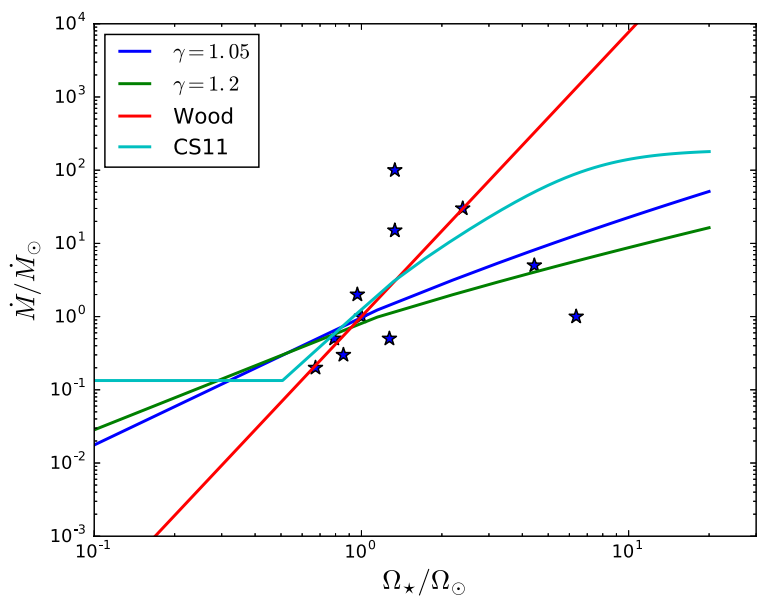

Fig. 1. Mass Loss model comparison with observations of Wood et al. (2005) represented by the blue stars. The model described here (Réville et al. 2016), yields a ideal mass loss shown in blue. The model of Holzwarth \& Jardine (2007) is shown in green, and the model of Cranmer \& Saar (2011) is shown in cyan. The fit proposed by Wood et al. (2005) is shown in red.

directions to transpose solar wind models in the stellar context (see Holzwarth \& Jardine 2007; Cranmer \& Saar 2011; Suzuki 2013). Our approach rely on the widely used polytropic wind model that can describe the acceleration of an outflow from a million Kelvin hot corona thanks to a modified adiabatic index $\gamma$ close to unity (see, e.g. Parker 1965; Weber \& Davis 1967; Mestel 1968; Washimi \& Shibata 1993; Keppens \& Goedbloed 1999). In the spherically symmetry purely hydro hypothesis, the model has three degrees of freedom, which can be reduced to the coronal base temperature and density and the polytropic index. Following the work of Ivanova \& Taam (2003) and Holzwarth \& Jardine (2007), we describe the evolution of the coronal parameters as power laws of the rotation rate:

$$
T=T_{\odot}\left(\frac{\Omega_{\star}}{\Omega_{\odot}}\right)^{0.1}, \quad n=n_{\odot}\left(\frac{\Omega_{\star}}{\Omega_{\odot}}\right)^{0.6},
$$

where $T_{\odot}=1.5 \times 10^{6} \mathrm{~K}$ and $n_{\odot}=10^{8} \mathrm{~cm}^{-3}$ are calibrated such that a wind with $\gamma=1.05$ recovers a wind velocity of $444 \mathrm{~km} \mathrm{~s}^{-1}$ at $1 \mathrm{AU}$ and a mass loss rate of $3.2 \times 10^{-14} M_{\odot} /$ yr. The value of $\gamma$ has been chosen accordingly to many older works, including ours (Matt et al. 2012; Réville et al. 2015a,b). This model yields a mass loss rates that can be computed as a function of $\Omega_{\star}$ and compared with observational data.

Figure 1 shows several models of mass loss rates and the scatter of observational points derived by the work of Wood et al. (2005). The law derived by our model is shown in blue, the green curve is the fiducial model of Holzwarth \& Jardine (2007), the cyan curve is from the model of Cranmer \& Saar (2011), and the red 
model is the fit of Wood et al. (2005), which does not consider the two most rightsided points, invoking a change of regimes for higher stellar activity. Although our model cannot account for the highest mass loss rates derived from some Ly $\alpha$ measurements, it describes smoothly the mass loss of very active stars without any transition, which remains difficult to explain (Vidotto et al. 2016).

We can then focus on the full MHD description of the stellar coronae of Zeeman Doppler Imaging (ZDI) targets (Semel 1989; Donati \& Brown 1997; Donati \& Landstreet 2009). In our latest study, Réville et al. (2016), we used several ZDI maps of the surface magnetic field of 5 young K-type stars (Folsom et al. 2016) and the Sun. We chose to study the evolution of the wind properties using stars with similar properties, except for their age that vary from $27 \mathrm{Myr}$ to the age of the Sun. We solve the ideal MHD equations using the PLUTO code (Mignone et al. 2007) in a cartesian box, constraining the surface magnetic field with the observed ZDI map.

Figure 2, shows the steady state obtained after the numerical resolution of the MHD equations. The thermal pressure gradient as well as magneto-centrifugal effect for fast rotators open field lines that were initially closed. Coronal loops remain where the magnetic tension is able to confine the coronal plasma. The Alfvén surface is shown in beige and stands for the place where the outflow reaches the Alfvén speed $V_{A}=B / \sqrt{4 \pi \rho}$. The Alfvén surface decrease with age and rotation rate mostly because of the decrease of the amplitude of the magnetic field. The magnetic topology does not change drastically as a significant dipole is always dominating the large scale coronal structure. This dipole is, however, strongly inclined in some cases making the 3D approach necessary.

\section{Evolution of angular momentum loss}

We find our simulations to comply fairly well with secular spin evolution models, where the angular momentum loss is generally expressed (Kawaler 1988; Bouvier et al. 1997) as

$$
\dot{J}=\dot{J}_{\odot}\left(\frac{\min \left(\Omega_{\star}, \Omega_{\mathrm{sat}}\right)}{\Omega_{\odot}}\right)^{2}\left(\frac{\Omega_{\star}}{\Omega_{\odot}}\right)\left(\frac{M_{\odot}}{M_{\star}} \frac{R_{\star}}{R_{\odot}}\right)^{0.5} .
$$

The saturation angular velocity $\Omega_{\text {sat }}$ represents the transition between the linear and the $\Omega_{\star}^{3}$ regime. In our sample, all stars more or less share the same mass and radius, and for K-type stars, the saturation is usually $\Omega_{\text {sat }}=8 \Omega_{\odot}$, so we can write

$$
\dot{J}=\dot{J}_{\odot} \frac{\tilde{\Omega}_{\star} \Omega_{\star}}{\Omega_{\star}^{3}},
$$

where $\tilde{\Omega}_{\star}=\min \left(\Omega_{\star}, 8 \Omega_{\odot}\right)$. In Figure 3 , we compare the angular momentum loss we obtain in our simulations (see Réville et al. 2016, for more details on its calculation), and the latter analytical formulation. In addition to the good global agreement, a saturation of the angular momentum loss naturally appears. It is important to insist here that no saturation is imposed in our simulations. 

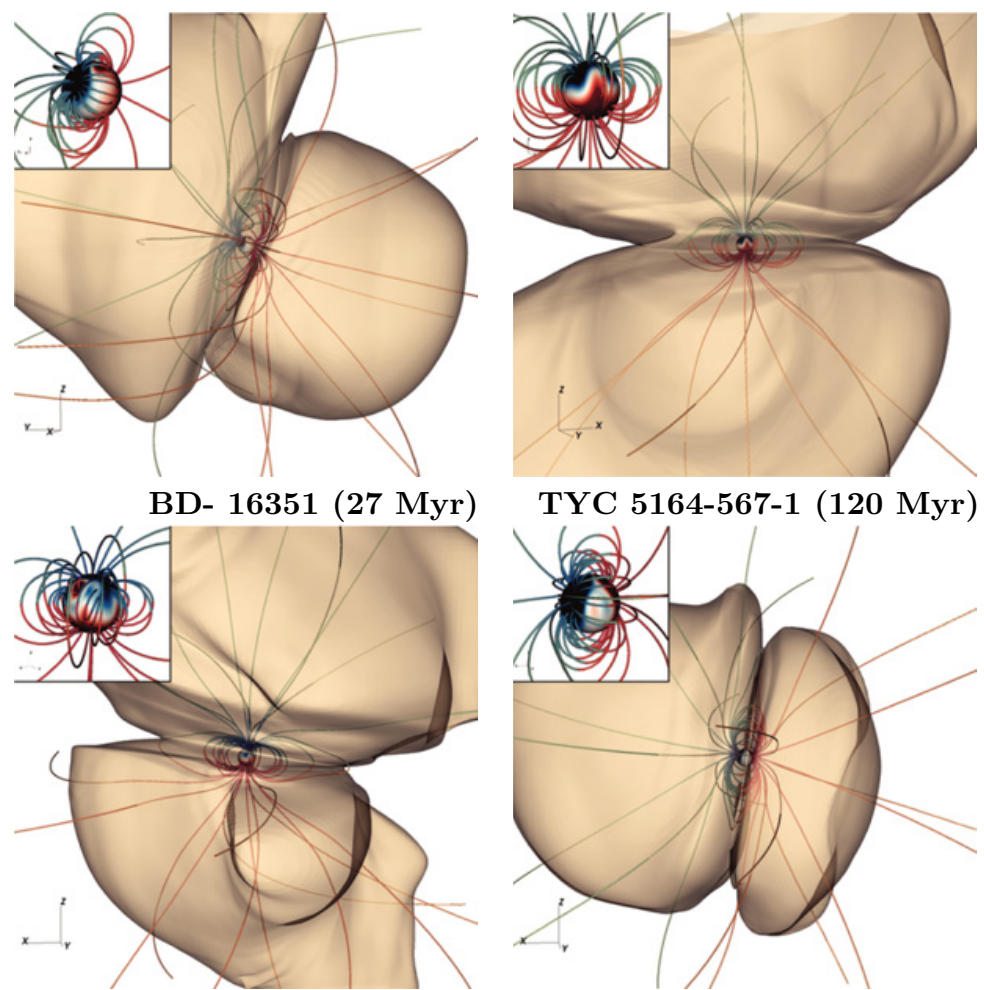

HII 296 (125 Myr)
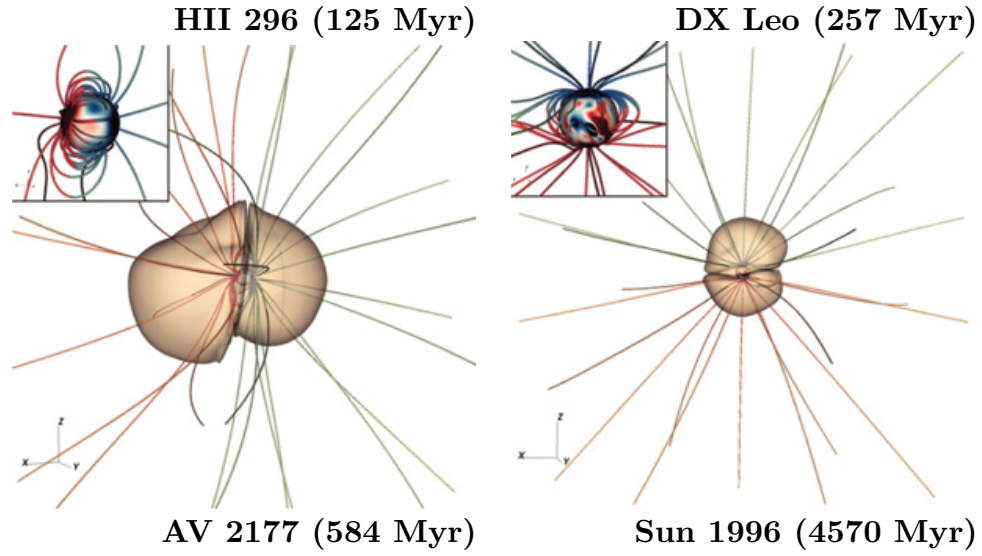

Fig. 2. Steady state of the 3D simulations. The Alfvén surfaces are shown in beige. The surface radial field is shown at the surface of the star and field lines of positive polarity are shown in red, negative polarity in blue. The Alfvén surfaces are largely shaped by the dominant dipole of each case. However, they are irregular due to the dipole inclination and higher order components of the magnetic field. The size of the Alfvén surface grows with the amplitude of the field. We show a zoom-in on the stellar surface with smaller scale features at the surface in the top left corner of each panel. 

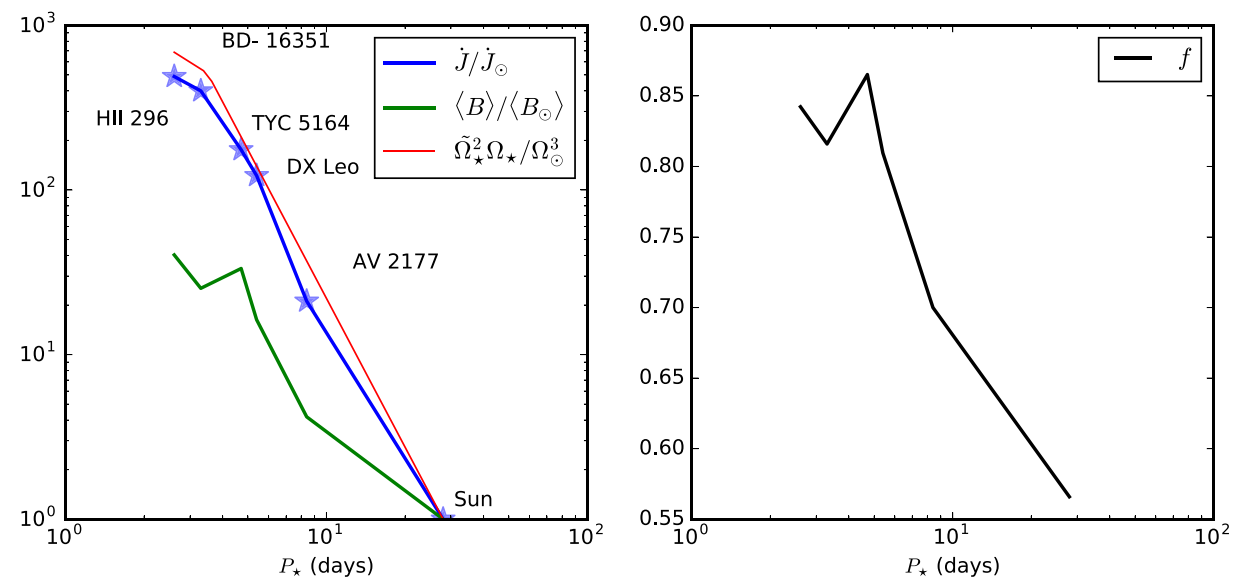

Fig. 3. In the left panel, evolution of mass and angular momentum loss in our six simulations (thick blue), compared to the relation 3.1 in red. The evolution of the magnetic field amplitude is shown in green. All quantities have been normalized to the solar value. The filling factor $f$ is shown on the right panel, we can see its value saturating close to 1 , its maximum value.

This behavior is the result of the competing mechanisms of the coronal model we described earlier and the prescription of the observed magnetic fields.

The amplitude of the magnetic field (the green line of Fig. 3), despite a slower pace for fast rotators, keeps increasing. The filling factor, that we define here as

$$
(1-f)=\frac{\Phi_{\text {open }}}{\Phi_{\star}}
$$

where $\Phi$ is the unsigned magnetic flux taken beyond all the closed coronal loops $\left(\Phi_{\text {open }}\right)$ or at the stellar surface $\left(\Phi_{\star}\right)$, becomes really close to 1 , its maximum value for the fastest rotators. In Réville et al. (2016), we interpreted the angular momentum loss saturation as a saturation of the mass loss, because of the very large coverage of closed loops on the stellar surface. More generally we can say that, as the AML is a growing function of both the open flux and the mass loss (Réville et al. 2015a), its saturation in the fast rotating regime is likely due to the very small surface coverage of open regions.

\section{Discussion}

The evolution of the angular momentum loss with rotation (or age) is controlled by the heating and accelerating processes of the wind and the dynamo processes that create the magnetic field in the convective envelope. Those two mechanisms are not independent and most likely possess a positive correlation. The energy necessary to accelerates the solar wind can be found in the Poynting flux at the 
photosphere, and is as such probably a growing function of the global field strength in other stars. Regarding the angular momentum loss, which can be embodied by the famous relationship of Weber \& Davis (1967): $\dot{J}=\Omega_{\star} \dot{M} R_{A}^{2}$, those two mechanisms seem to both push in the same direction in the non-saturated regime. In our model, we find that The Aflvén increases with the rotation rate as well as the mass loss. The saturation comes when a competition arises between the two phenomena. Large scale magnetic loops cover so much of the stellar surface that not enough material can escape to compensate.

This interpretation is not new, and the filling factor has been often involved in explaining saturation. Nonetheless, this work allows a discussion that includes the magnetic field geometry. Our saturation scenario has, by construction, the advantage of being coherent with observations, where large scale, dipolar components of the magnetic field are controlling the braking. Moreover, as X-ray luminosity is higher in smaller, denser, hotter loops, sudden change from large scale to small scale component would imply an even stronger increase of the X-ray flux. On the contrary, when large scale components are conserved, the smaller loops ${ }^{1}$, protected from the wind inside the coronal streamers are free to develop until no space is left on the stellar surface, which produces the saturation.

Our model represents only a small sample of stars, and even faster rotators would need to be simulated (the simulation cost increases with the strength of the magnetic field). This result also strongly depends on the exponent chosen in the $(T, n)$ laws, and more generally on how the acceleration of stellar winds depends on stellar properties. We aim to develop more physically motivated model of stellar wind acceleration (in line with Cranmer \& Saar 2011; Suzuki 2013), that should be coupled with dynamo theory.

\section{References}

Barnes, S., \& Sofia, S., 1996, ApJ, 462, 746

Barnes, S.A., 2003, ApJ, 586, 464

Bouvier, J., Forestini, M., \& Allain, S., 1997, A\&A, 326, 1023

Brown, T.M., 2014, ApJ, 789, 101

Brun, A.S., Browning, M.K., Dikpati, M., Hotta, H., \& Strugarek, A., 2013, Space Sci. Rev.

Cranmer, S.R., \& Saar, S.H., 2011, ApJ, 741, 54

Donati, J.-F., \& Brown, S.F., 1997, A\&A, 326, 1135

Donati, J.-F., \& Landstreet, J.D., 2009, ARA\&A, 47, 333

Folsom, C.P., Petit, P., Bouvier, J., et al., 2016, MNRAS, 457, 580

Garraffo, C., Drake, J.J., \& Cohen, O., 2015, ApJ, 807, L6

Holzwarth, V., \& Jardine, M., 2007, A\&A, 463, 11

Ivanova, N., \& Taam, R.E., 2003, ApJ, 599, 516

1 That cannot be detected by Zeeman Doppler Imaging, where the maximum resolution is around $\ell=8$ (Folsom et al. 2016). 
Kawaler, S.D., 1988, Astrophys. J., 333, 236

Keppens, R., \& Goedbloed, J.P., 1999, A\&A, 343, 251

Matt, S.P., Brun, A.S., Baraffe, I., Bouvier, J., \& Chabrier, G., 2015, ApJ, 799, L23

Matt, S.P., Pinzón, G., Greene, T.P., \& Pudritz, R.E., 2012, ApJ, 745, 101

Mestel, L., 1968, MNRAS, 138, 359

Mignone, A., Bodo, G., Massaglia, S., et al., 2007, ApJS, 170, 228

Noyes, R.W., Weiss, N.O., \& Vaughan, A.H., 1984, ApJ, 287, 769

Parker, E.N., 1965, Space Sci. Rev., 4, 666

Pizzolato, N., Maggio, A., Micela, G., Sciortino, S., \& Ventura, P., 2003, A\&A, 397, 147

Réville, V., Brun, A.S., Matt, S.P., Strugarek, A., \& Pinto, R.F., 2015a, ApJ, 798, 116

Réville, V., Brun, A.S., Strugarek, A., et al., 2015b, ApJ, 814, 99

Réville, V., Folsom, C.P., Strugarek, A., \& Brun, A.S., 2016, ApJ, 832, 145

Rosner, R., Tucker, W.H., \& Vaiana, G.S., 1978, ApJ, 220, 643

Semel, M., 1989, A\&A, 225, 456

Suzuki, T.K., 2013, Astronomische Nachrichten, 334, 81

Vidotto, A.A., Donati, J.-F., Jardine, M., et al., 2016, MNRAS, 455, L52

Washimi, H., \& Shibata, S., 1993, MNRAS, 262, 936

Weber, E.J., \& Davis, Jr., L., 1967, ApJ, 148, 217

Wood, B.E., Müller, H.-R., Zank, G.P., Linsky, J.L., \& Redfield, S., 2005, ApJ, 628, L143 